Pacific Journal of Mathematic 


\section{VIBRATION OF A NONHOMOGENEOUS MEMBRANE}

\section{H. Protter}

1. Introduction. We consider a simply connected two dimensional domain $D$ with a nonhomogeneous membrane $M$ stretched across $D$ and fixed at the boundary $\Gamma$. Let $p(x, y) \geq 0$ be the density function of the membrane. We shall be concerned with the first eigenvalue $\lambda_{0}$ of the equation

$$
u_{x x}+u_{y y}+\lambda p(x, y) u=0
$$

subject to the condition $u=0$ on $\Gamma$. Let $K$ be the circle with boundary $C$ on which a homogeneous membrane $M_{1}$ of the same mass as $M$ is stretched. Let $\lambda_{1}$ be the first eigenvalue of

$$
v_{x x}+v_{y y}+\lambda v=0
$$

with $v=0$ on $C$. In a recent paper Nehari [1] established the following interesting result.

Theorem. (Nehari) If $\log p(x, y)$ is subharmonic then

$$
\lambda_{0} \geq \lambda_{1} \text {. }
$$

Nehari further showed that relaxation to the condition that $p(x, y)$ be subharmonic is not possible. In fact for the case that $D$ is a circle and $p(x, y)$ is superharmonic the inequality in (3) is shown to be reversed.

It is the purpose of this paper to establish comparison theorems for the first eigenvalue of homogeneous and nonhomogeneous membranes of the same shape. That is, we shall consider the first eigenvalue of equations (1) and (2) in the same domain $D$ subject to the boundary condition $u=0$ and $v=0$ on $\Gamma$ respectively. We denote the first eigenvalue of the latter problem by $\mu$ and consider comparisons between $\lambda_{0}$ and $\mu$. We of course have the completely trivial comparison

$$
\lambda_{0} \geq \mu
$$

if $0 \leq p(x, y) \leq 1$ throughout $D$. Nehari's result pertained to the case where $p(x, y)$ had average value 1 and thus we wish to obtain relations between $\lambda_{0}$ and $\mu$ for density functions which may become large.

A general technique for obtaining lower bounds for the first eigenvalue for a homogeneous membrane in a domain $D$ follows from the

Received March 12, 1959. This research was supported by the United States Air Force through the Air Force Office of Scientific Research of the Air Research and Development Command under Contract No. AF 49 (638)-398. 
inclusion principle. If $D$ is contained in $D_{0}$ then the first eigenvalue for $D$ is larger than that for $D_{0}$. If $D$ is bounded then we can enclose $D$ in a rectangle or circle for which the first eigenvalue is known. This technique is also possible for nonhomogeneous membranes as will be readily seen from the basic inequalities established in $\S 2$. In $\S 3$ comparison theorems are established when the density function is assumed to satisfy various conditions involving the behavior of the second derivative of $p(x, y)$. Section 4 discusses comparison theorems between two nonhomogeneous membranes.

2. Basic inequalities. Let $u$ be any function which vanishes on $\Gamma$, and let $a(x, y)$ be an arbitrary $C^{2}$ function in $D$. We apply Green's theorem to the expression

$$
\iint_{D} a u\left(u_{x x}+u_{y y}\right) d x d y
$$

and obtain

(4) $\iint_{D} a u\left(u_{x x}+u_{y y}\right) d x d y=-\iint_{D} a\left(u_{x}^{2}+u_{y}^{2}\right) d x d y+\frac{1}{2} \iint_{D} u^{2}\left(a_{x x}+a_{y y}\right) d x d y$

The boundary integrals vanishing in virtue of $u=0$ on $\Gamma$. Further we let $P(x, y), Q(x, y)$ be arbitrary $C^{\prime}$ functions in $D$ and note that

$$
\left.\iint\left[P u^{2}\right)_{x}+\left(Q u^{2}\right)_{y}\right] d x d y=0 .
$$

Performing the differentiations in (5) and adding the result to (4) we get

$$
\begin{aligned}
& -\iint_{D} a u\left(u_{x x}+u_{y y}\right) d x d y \\
& =+\iint_{D}\left\{a\left(u_{x}^{2}+u_{y}^{2}\right)+2 P u u_{x}+2 Q u u_{y}\right. \\
& \left.\quad+\left[P_{x}+Q_{y}-\frac{1}{2}\left(a_{x x}+a_{y y}\right)\right] u^{2}\right\} d x d y .
\end{aligned}
$$

If $u$ were the first eigenfunction and $\lambda$ the first eigenvalue of the nonhomogeneous membrane, then (1) would hold and the above expression would be

$$
\begin{aligned}
& \iint_{D}\left\{a\left(u_{x}^{2}+u_{y}^{2}\right)+2 P u u_{x}+2 Q u u_{y}\right. \\
& \left.\quad+\left[P_{x}+Q_{y}-\frac{1}{2}\left(a_{x x}+a_{y y}\right)-a \lambda p\right] u^{2}\right\} d x d y=0
\end{aligned}
$$


On the other hand this integrand is a quadratic form in $u_{x}, u_{y}, u$. It will be a positive definite form if $a>0$ and

$$
P_{x}+Q_{y} \geq \frac{1}{a}\left(P^{2}+Q^{2}\right)+\frac{1}{2}\left(a_{x x}+a_{y y}\right)+a p \lambda .
$$

If $a, P, Q, \lambda$ happen to satisfy (7) then clearly it is impossible that (6) holds. However if (7) holds for any value $\bar{\lambda}$, it obviously holds for $0 \leq \lambda \leq \bar{\lambda}$ and thus (6) cannnot hold for any function $u(x, y)$ with $0 \leq \lambda \leq \bar{\lambda}$. This implies that $\bar{\lambda}$ is a lower bound for the first eigenvalue of (1).

We shall therefore be concerned with the possibility of selection of functions $P, Q, a$ such that inequality (7) holds for some value $\bar{\lambda}$. For convenience we assume the bounded domain $D$ is in the first quadrant. We select the function $a(x, y)$ to be

$$
a(x, y)=\sin \alpha x \sin \beta x
$$

where $\alpha$ and $\beta$ are constants selected so that $a(x, y)$ is positive throughout $\bar{D}$. We define the quantities

$$
m_{0}=\min _{\bar{\nu}} \alpha
$$

and $M_{0}=m_{0}^{-1}$. Inequality (7) is implied by the inequality

$$
P_{x}+Q_{y} \geq M_{0}\left(P^{2}+Q^{2}\right)+\frac{1}{2}\left(a_{x x}+a_{y y}\right)+a p \lambda
$$

and if we define

$$
P_{1}=M_{0} P, Q_{1}=M_{0} Q
$$

(8) is equivalent to

$$
P_{1 x}+Q_{1 y} \geq P_{1}^{2}+Q_{1}^{2}+\frac{1}{2} M_{0}\left(a_{x x}+a_{y y}\right)+M_{0} a p \lambda .
$$

Let $\phi(x, y)$ be the first eigenfunction for equation (2) in the domain $D$ subject to the condition $v=0$ on $\Gamma$. That is,

$$
\phi_{x x}+\phi_{y y}+\mu \phi=0 \text {. }
$$

We make the following selection:

$$
P_{1}=-\frac{\phi_{x}}{\phi}, \quad Q_{1}=-\frac{\phi_{y}}{\phi}
$$


and obtain from (9)

$$
\mu \geq M_{0} \sin \alpha x \sin \beta y\left[-\frac{1}{2}\left(\alpha^{2}+\beta^{2}\right)+\lambda p(x, y)\right]
$$

Define the quantity

$$
N_{0}=\max _{\bar{D}} p(x, y) \sin \alpha x \sin \beta y
$$

and we obtain the following result.

THEOREM 1. Let $\lambda_{0}$ be the first eigenvalue for the nonhomogeneous membrane with density function $p(x, y)$ spanning a domain $D$ and $\mu$ the first eigenvalue for the homogeneous membrane spanning the same domain. Then

$$
\lambda_{0} \geq \frac{\mu+\frac{1}{2}\left(\alpha^{2}+\beta^{2}\right)}{M_{0} N_{0}} .
$$

The theorem is an immediate consequence of inequality (10) which exhibits the positive definiteness of the integrand (6). Inequality (11) is a statement that (10) must be violated.

We note that (11) is a useful relation if $N_{0}$ is particularly small; hence this states that $p(x, y)$ should be small near the center of the membrane, but may be large near the outer edge and still (11) will be a significant lower bound for $\lambda_{0}$. The basic distinction between (11) and other results lies in the fact that $p(x, y)$ has no restriction except positivity.

A word should be said about the selection of the function $a(x, y)$. We chose for this function the first eigenfunction for the equation (2) applied to a rectangle which contains $D$ in its interior. We could have selected for $a(x, y)$ the first eigenfunction for any including domain, e.g., a circle, equilateral triangle, etc. with a resulting inequality similar to (11). Finally the selection $a \equiv 1$ yields the standard result

$$
\lambda_{0} \geq \frac{\mu}{\max _{\bar{D}} p(x, y)}
$$

3. Bounds with condition on the density function. We return to inequality (7) and the selection of $a, P$, and $Q$. We recall that these functions may be arbitrary except that $a(x, y)$ must be positive. We make the choice

$$
a(x, y)=\frac{1}{p(x, y)}
$$


Then (7) becomes

$$
P_{x}+Q_{y} \geq p(x, y)\left(P^{2}+Q^{2}\right)+\frac{1}{2} \Delta\left(\frac{1}{p}\right)+\lambda .
$$

We define

$$
p_{0}=\max _{\bar{D}} p(x, y)
$$

and select

$$
P=-\frac{\phi_{x}}{p_{0} \phi}, \quad Q=-\frac{\phi_{y}}{p_{0} \phi}
$$

where, as before, $\phi$ is the first eigenfunction of (2) for the domain $D$. We obtain

$$
\frac{\mu}{p_{0}} \geq \frac{1}{2} \Delta\left(\frac{1}{p}\right)+\lambda
$$

If we assume the function $1 / p$ is superharmonic and set

$$
N_{1}=-\max _{\bar{D}} \frac{1}{2} \Delta\left(\frac{1}{p}\right)
$$

we obtain the following result.

THEOREM 2. Let $\lambda_{0}$ be the first eigenvalue for the nonhomogeneous membrane with density function $p(x, y)$ and $\mu$ the corresponding first eigenvalue for the homogeneous membrane spanning the same domain $D$. If $1 / p$ is superharmonic in $D$ we have the inequality

$$
\lambda_{0} \geq \frac{\mu}{p_{0}}+N_{1}
$$

where $p_{0}$ and $N_{1}$ are given by (13) and (14) respectively.

It is possible to obtain a comparison theorem for the case where $\log p$ is subharmonic. To see this we make the choice

$$
a(x, y)=\log \frac{1}{p}
$$

and we assume $0<p(x, y)<1$ in $\bar{D}$. With this selection we take

$$
P=-\frac{\phi_{x}}{p_{0} \phi}, \quad Q=-\frac{\phi_{y}}{p_{0} \phi}
$$


as before and obtain

$$
\frac{\mu}{p_{0}} \geq \frac{1}{2} \Delta\left(\log \frac{1}{p}\right)+\lambda p \log \frac{1}{p} .
$$

We assume $\log p$ is subharmonic and define

$$
\begin{aligned}
& N_{2}=\frac{1}{2} \min _{\bar{D}} \Delta(\log p) \\
& N_{3}=\max _{\bar{D}} p \log \frac{1}{p} .
\end{aligned}
$$

THEOREM 3. Let $\lambda_{0}$ and $\mu$ be as in Theorem 2. If $\log p$ is subharmonic in $D$ then

$$
\lambda_{0} \geq \frac{\mu}{p_{0} N_{3}}+\frac{N_{2}}{N_{3}}
$$

where $N_{2}$ and $N_{3}$ are given by (16) and (17).

A final application of this type which we exhibit results from the selection

$$
a=e^{\alpha p(x, y)}
$$

where $\alpha$ is a constant which remains to be chosen. If we suppose that $p$ is strictly superharmonic and select $\alpha$ so that

$$
\frac{1}{2} \Delta p+\alpha\left(p_{x}^{2}+p_{y}^{2}\right) \leq 0
$$

we obtain the relation

$$
\lambda_{0} \geq \mu \max _{\bar{D}}\left(\frac{e^{-\alpha p}}{p}\right)
$$

4. Comparison of two nonhomogeneous membranes. Let $q(x, y)$ be a second density function corresponding to a membrane spanning $D$ and let $\nu$ be the first eigenvalue for

$$
w_{x x}+w_{y y}+\nu q(x, y) w=0
$$

with boundary condition $w=0$ on $\Gamma$. We denote the corresponding first eigenfunction by $\psi(x, y)$. It is possible to compare $\lambda_{0}$ and $\nu$ when the functions $p$ and $q$ satisfy various relations. Let

$$
q_{0}=\max _{\bar{D}} q(x, y)
$$


$(20)$

$$
r_{0}=\max _{\bar{\nu}} \frac{p(x, y)}{q(x, y)}
$$

and

$$
N_{4}=-\max _{\bar{\nu}} \Delta\left(\frac{q}{p}\right)
$$

We make the selections

$$
a=\frac{q}{p}, \quad P=-\frac{\psi_{x}}{r_{0} \psi}, \quad Q=-\frac{\psi_{y}}{r_{0} \psi}
$$

and find

$$
\frac{\nu q}{r_{0}} \geq \frac{5}{2} \Delta\left(\frac{q}{p}\right)+q \lambda
$$

THEOREM 4. Let $\lambda_{0}$ and $\nu$ be the first eigenvalue corresponding to density functions $p$ and $q$ respectively. If $q / p$ is superharmonic then we have the inequality

$$
\lambda_{0} \geq \frac{\nu}{r_{0}}+\frac{1}{2} \frac{N_{4}}{q_{0}}
$$

where $q_{0}, r_{0}$ and $N_{4}$ are given by (19), (20) and (21).

Additional inequalities, analogous to those obtained in $\S \S 2$ and 3 may be obtained by other selections for $a, P$ and $Q$.

\section{BIBLIOGRAPHY}

1. Z. Nehari, On the principal frequency of a membrane, Pacific J. Math. 8 (1958), 285-293. UNIVERSITY OF CALIFORNIA

BERKELEY 



\section{PACIFIC JOURNAL OF MATHEMATICS}

EDITORS

David Gilbarg

Stanford University

Stanford, California

R. A. Beaumont

University of Washington

Seattle 5 , Washington
A. L. Whiteman

University of Southern California

Los Angeles 7, California

L. J. PAIGE

University of California

Los Angeles 24, California

\section{ASSOCIATE EDITORS}

\author{
E. F. BECKENBACH \\ C. E. BURGESS \\ E. HEWITT \\ A. HORN
}

\author{
V. GANAPATHY IYER \\ R. D. JAMES \\ M. S. KNEBELMAN \\ L. NACHBIN
}
I. NIVEN
E. G. STRAUS
T. G. OSTROM
G. SZEKERES
H. L. ROYDEN
F. WOLF
M. M. SCHIFFER
K. YOSIDA

\section{SUPPORTING INSTITUTIONS}

\author{
UNIVERSITY OF BRITISH COLUMBIA \\ CALIFORNIA INSTITUTE OF TECHNOLOGY \\ UNIVERSITY OF CALIFORNIA \\ MONTANA STATE UNIVERSITY \\ UNIVERSITY OF NEVADA \\ OREGON STATE COLLEGE \\ UNIVERSITY OF OREGON \\ OSAKA UNIVERSITY \\ UNIVERSITY OF SOUTHERN CALIFORNIA
}

\author{
STANFORD UNIVERSITY \\ UNIVERSITY OF TOKYO \\ UNIVERSITY OF UTAH \\ WASHINGTON STATE COLLEGE \\ UNIVERSITY OF WASHINGTON \\ $* \quad * \quad *$ \\ AMERICAN MATHEMATICAL SOCIETY \\ CALIFORNIA RESEARCH CORPORATION \\ HUGHES AIRCRAFT COMPANY \\ SPACE TECHNOLOGY LABORATORIES
}

Mathematical papers intended for publication in the Pacific Journal of Mathematics should be typewritten (double spaced), and the author should keep a complete copy. Manuscripts may be sent to any one of the four editors. All other communications to the editors should be addressed to the managing editor, L. J. Paige at the University of California, Los Angeles 24, California.

50 reprints per author of each article are furnished free of charge; additional copies may be obtained at cost in multiples of 50 .

The Pacific Journal of Mathematics is published quarterly, in March, June, September, and December. The price per volume (4 numbers) is $\$ 12.00$; single issues, $\$ 3.50$. Back numbers are available. Special price to individual faculty members of supporting institutions and to individual members of the American Mathematical Society: $\$ 4.00$ per volume; single issues, $\$ 1.25$.

Subscriptions, orders for back numbers, and changes of address should be sent to Pacific Journal of Mathematics, 2120 Oxford Street, Berkeley 4, California.

Printed at Kokusai Bunken Insatsusha (International Academic Printing Co., Ltd.), No. 6, 2-chome, Fujimi-cho, Chiyoda-ku, Tokyo, Japan.

PUBLISHED BY PACIFIC JOURNAL OF MATHEMATICS, A NON-PROFIT CORPORATION

The Supporting Institutions listed above contribute to the cost of publication of this Journal, but they are not owners or publishers and have no responsibility for its content or policies. 


\section{Pacific Journal of Mathematics}

\section{Vol. 9, No. 4 \\ August, 1959}

Frank Herbert Brownell, III, A note on Kato's uniqueness criterion for

Schrödinger operator self-adjoint extensions ............... 953

Edmond Darrell Cashwell and C. J. Everett, The ring of number-theoretic

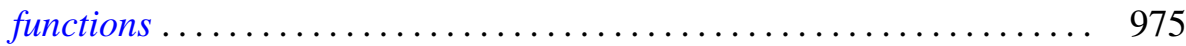

Heinz Otto Cordes, On continuation of boundary values for partial

differential operators ............................. 987

Philip C. Curtis, Jr., n-parameter families and best approximation . . . . . . 1013

Uri Fixman, Problems in spectral operators . . . . . . . . . . . . . . . 1029

I. S. Gál, Uniformizable spaces with a unique structure .............. 1053

John Mitchell Gary, Higher dimensional cyclic elements ............ 1061

Richard P. Gosselin, On Diophantine approximation and trigonometric

polynomials ..................................... 1071

Gilbert Helmberg, Generating sets of elements in compact groups ........ 1083

Daniel R. Hughes and John Griggs Thompson, The H-problem and the

structure of $H$-groups .................................. 1097

James Patrick Jans, Projective injective modules ................. 1103

Samuel Karlin and James L. McGregor, Coincidence properties of birth and

death processes ..................................... 1109

Samuel Karlin and James L. McGregor, Coincidence probabilities ........ 1141

J. L. Kelley, Measures on Boolean algebras ................... 1165

John G. Kemeny, Generalized random variables ................... 1179

Donald G. Malm, Concerning the cohomology ring of a sphere bundle ... . . 1191

Marvin David Marcus and Benjamin Nelson Moyls, Transformations on

tensor product spaces .................................. 1215

Charles Alan McCarthy, The nilpotent part of a spectral operator ........ 1223

Kotaro Oikawa, On a criterion for the weakness of an ideal boundary

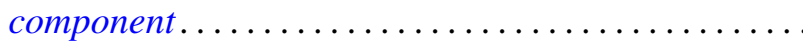

Barrett O'Neill, An algebraic criterion for immersion . . ............... 1239

Murray Harold Protter, Vibration of a nonhomogeneous membrane ... . . . . 1249

Victor Lenard Shapiro, Intrinsic operators in three-space . . . . . . . . . . . 1257

Morgan Ward, Tests for primality based on Sylvester's cyclotomic

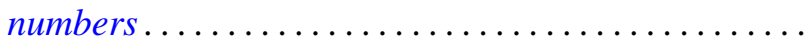

L. E. Ward, A fixed point theorem for chained spaces ....

Alfred B. Willcox, Šilov type $C$ algebras over a connected locally compact

abelian group..................................... 1279

Jacob Feldman, Correction to "Equivalence and perpendicularity of

Gaussian processes" ........................ 